\title{
ASPECTS CONCERNING THE ELECTRICAL Behaviour of Metalic Particle REINFORCED POLYMERIC COMPOSITE MATERIALS
}

\author{
Motoc LucA, D. \& OlTEAN, D.I.
}

Abstract: The paper approaches the eletrical properties and its variations under different loading conditions in case of particle reinfocerd polymeric composite materials. Different measurement methods and several material combinations will be investigated.

Key words: particle, metallic, polymeric, properties, electrical
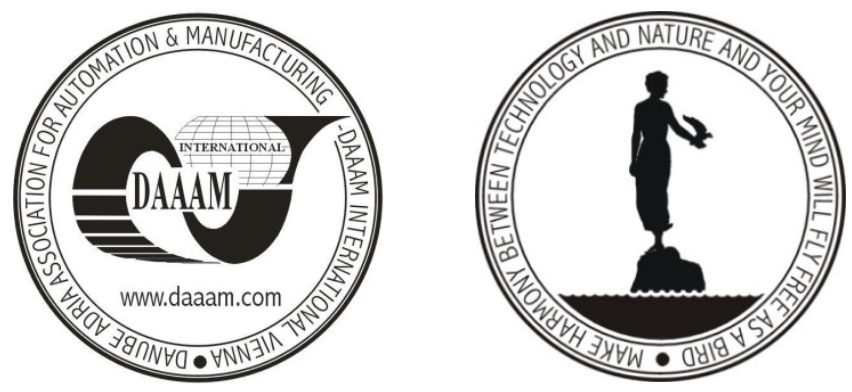

Authors' data: Dr. Ass. Prof. Motoc Luca, D[ana]; Oltean, D[anut], Transilvania University of Brasov, 29 Eroilor Av., 500107, Brasov, RO, danaluca@unitbv.ro, olteanu@unitbv.ro

This Publication has to be referred as: Motoc Luca, D[ana] \& Oltean, D[anut] I[oan] (2008). Aspects Concerning the Electrical Behaviour of Metalic Particle Reinforced Polymeric Composite Materials, Chapter 46 in DAAAM International Scientific Book 2008, pp. 543-550, B. Katalinic (Ed.), Published by DAAAM International, ISBN 978-3-901509-66-7, ISSN 1726-9687, Vienna, Austria DOI: $10.2507 /$ daaam.scibook.2008.46 